\title{
Heat shock protein 90 and calcineurin pathway inhibitors enhance the efficacy of triazoles against Scedosporium prolificans via induction of apoptosis
}

\author{
Fazal Shirazi and Dimitrios P. Kontoyiannis* \\ Department of Infectious Diseases, Infection Control and Employee Health, The University of Texas M.D. Anderson Cancer Center, \\ Houston, TX 77030, U.S.A. \\ * Corresponding Author: Dimitrios P. Kontoyiannis, MD, ScD, Department of Infectious Diseases, Infection Control and Employee \\ Health, Unit 402, The University of Texas MD Anderson Cancer Center, 1515 Holcombe Boulevard; Houston, TX 77030, USA; Tel: +1 \\ 713792 6237; Fax: +1 713745 6839; Email: dkontoyi@mdanderson.org
}

\begin{abstract}
Scedosporium prolificans is a pathogenic mold resistant to current antifungals, and infection results in high mortality. Simultaneous targeting of both ergosterol biosynthesis and heat shock protein 90 (Hsp90) or the calcineurin pathway in S. prolificans may be an important strategy for enhancing the potency of antifungal agents. We hypothesized that the inactive triazoles posaconazole (PCZ) and itraconazole (ICZ) acquire fungicidal activity when combined with the calcineurin inhibitor tacrolimus (TCR) or Hsp90 inhibitor 17-demethoxy-17-(2-propenylamino) geldanamycin (17AAG). PCZ, ICZ, TCR and 17AAG alone were inactive in vitro against $S$. prolificans spores (MICs > $128 \mu \mathrm{g} / \mathrm{ml}$ ). In contrast, MICs for PCZ or ICZ in combination with TCR or 17AAG $(0.125-0.50 \mu \mathrm{g} / \mathrm{ml})$ were much lower compared with drug alone. In addition PCZ and ICZ in combination with TCR or 17AAG became fungicidal. Because apoptosis is regulated by the calcineurin pathway in fungi and is under the control of Hsp90, we hypothesized that this synergistic fungicidal effect is mediated via apoptosis. This observed fungicidal activity was mediated by increased apoptosis of $S$. prolificans germlings, as evidenced by reactive oxygen species accumulation, decreased mitochondrial membrane potential, phosphatidylserine externalization, and DNA fragmentation. Furthermore, induction of caspase-like activity was correlated with TCR or 17AAG + PCZ/ICZ-induced cell death. In conclusion, we report for the first time that PCZ or ICZ in combination with TCR or 17AAG renders $S$. prolificans exquisitely sensitive to PCZ or ICZ via apoptosis. This finding may stimulate the development of new therapeutic strategies for patients infected with this recalcitrant fungus.
\end{abstract}

doi: $10.15698 /$ mic2014.06.150 Received originally: 12.03.2014; in revised form: 13.05.2014, Accepted 20.05.2014 Published 02.06.2014.

Keywords: apoptosis, $17 A A G$, calcineurin, itraconazole, posaconazole, reactive oxygen species.

\section{INTRODUCTION}

Scedosporium prolificans is an emerging filamentous fungus that causes severe, frequently fatal pulmonary or disseminated opportunistic infections in immunocompromised patients [1]. S. prolificans is inherently resistant to treatment with a wide range of antifungals, including the new generation of broad-spectrum triazoles [1-4]. Hence, new therapeutic strategies for Scedosporium infections are urgently needed.

In pathogenic fungi, the calcineurin pathway and heat shock protein 90 (Hsp90) play major roles in maintaining fungal homeostatic cell responses, including resistance to antifungal agents [5-10]. The calcineurin inhibitor tacrolimus (TCR) is an immunosuppressive agent widely used in solid organ and hematopoietic stem cell transplant recipients to prevent graft rejection [11]. TCR binds to the intracellular protein immunophilin FKB12 and forms a complex, thereby inhibiting activation of the calcineurin pathway. In vitro studies have suggested synergy between triazoles and calcineurin inhibitors against Aspergillus spp. and the Mucorales [12-14]. Our group recently reported that treatment with the combination of TCR and posaconazole (PCZ) 
A
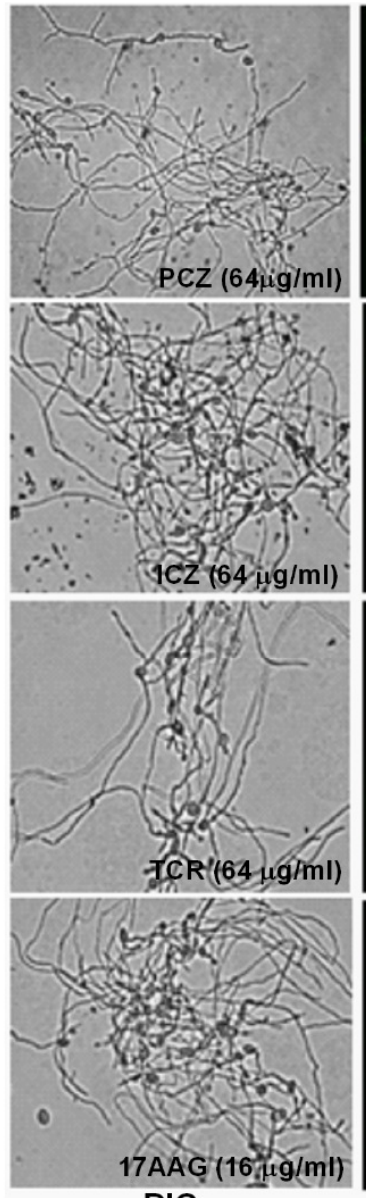

DIC

B

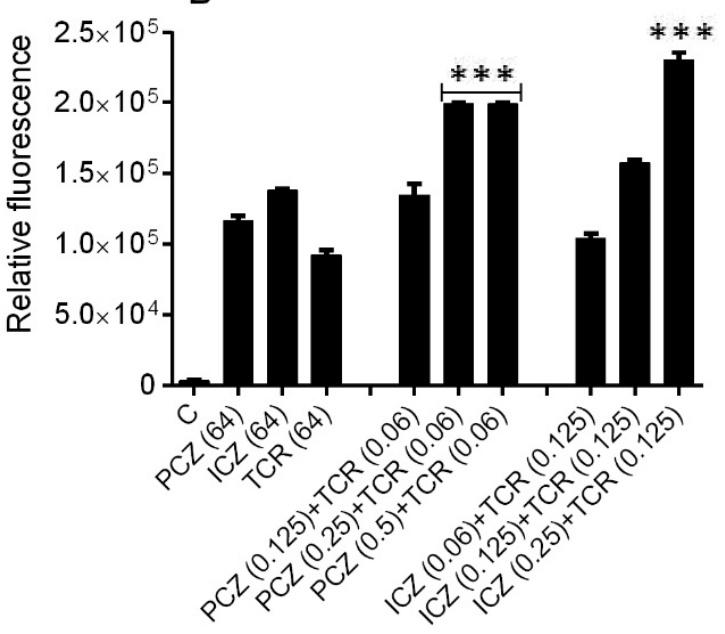

Antifungal Concentration $(\mu \mathrm{g} / \mathrm{ml})$

DiBAC
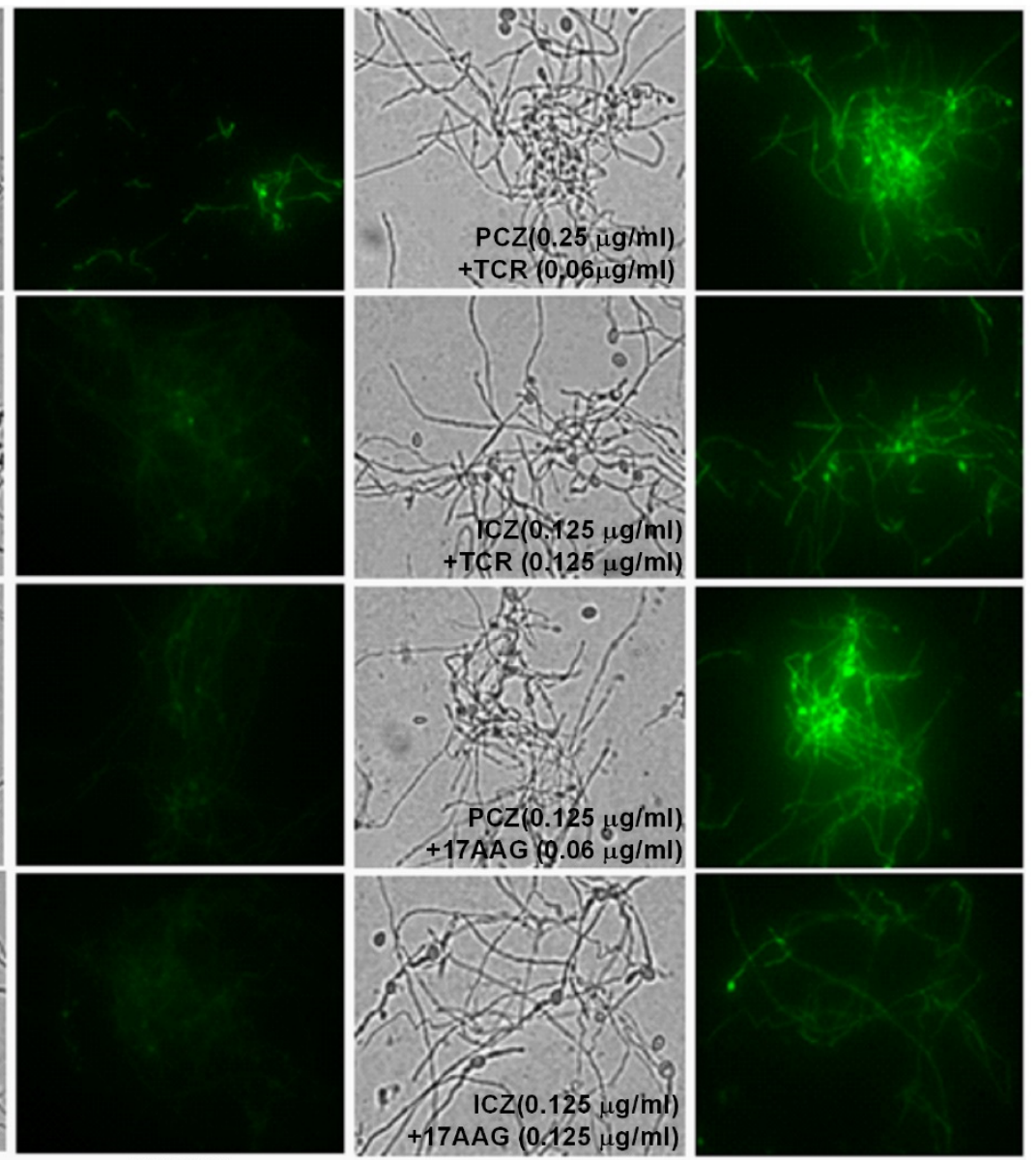

C

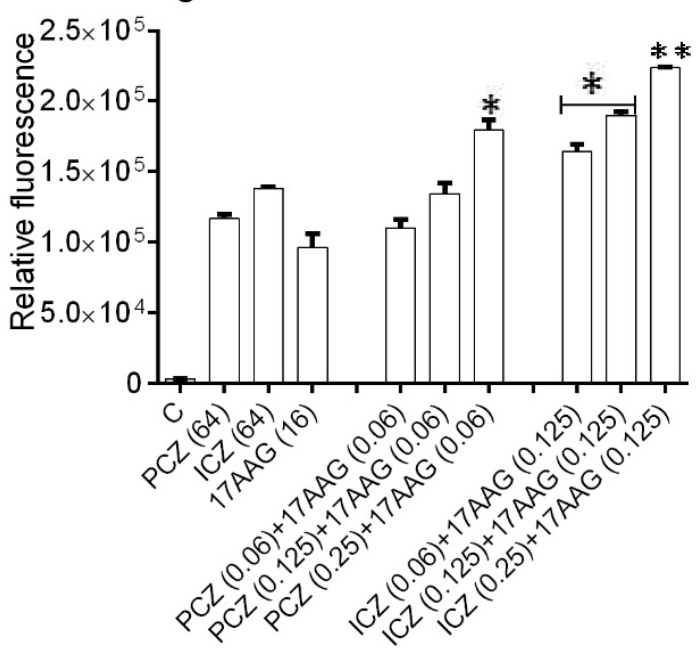

Antifungal Concentration $(\mu \mathrm{g} / \mathrm{ml})$

FIGURE 1: Fungicidal action of PCZ and ICZ alone and in combination with TCR and 17AAG against S. prolificans germlings (isolate 1). (A) Fluorescent images of $S$. prolificans germlings stained with the morbidity dye DiBAC. (B, C) Relative fluorescence levels in S. prolificans germlings treated with PCZ or ICZ plus TCR (B) or 17AAG (C) as determined using DiBAC staining. The experiments were performed in triplicate and repeated three times. ${ }^{*} p<0.05 ;{ }^{* *} p<0.001 ;{ }^{* * *} p<0.0001$ (compared with untreated control germlings and germlings exposed to drug alone). Error bars on graphs indicate standard deviation. DIC, differential interference contrast. 
improves control of invasive, necrotizing cutaneous mucormycosis in immunosuppressed mice compared with PCZ alone [15].

Hsp90 is a molecular chaperone involved in stress responses of Candida albicans and Aspergillus spp. and plays a major role in echinocandin resistance via regulation of the calcineurin pathway $[6,16]$. Specifically, pharmacological inhibition of Hsp90 by 17-demethoxy-17-(2propenylamino) geldanamycin (17AAG) prevents azole resistance and abrogates this resistance in $C$. albicans and A. fumigatus in a human host $[6,16]$. In addition, researchers recently suggested a role for the calcineurin pathway in regulation of apoptosis in fungi $[17,18]$. However, the role of Hsp90 in apoptosis remains unclear. Therefore, simultaneous targeting of both ergosterol biosynthesis and Hsp90 or calcineurin pathways in S. prolificans may be an important strategy for restoring the potency of antifungal agents. Specifically, we hypothesized that TCR or 17AAG in combination with the triazoles PCZ or itraconazole (ICZ) induces apoptosis in S. prolificans. Thus, we examined the effects of TCR and 17AAG co-administration on PCZ and ICZ activity using several in vitro methods to evaluate induction of apoptosis in S. prolificans.

\section{RESULTS}

PCZ and ICZ are inactive when used alone against S. prolificans, but exhibit significant fungicidal activity when combined with TCR or 17AAG

Individually, PCZ, ICZ, TCR, and 17AAG were inactive against $S$. prolificans (isolates 1 to 3 ), with minimum inhibitory concentrations (MICs) ranging from 32 to $128 \mu \mathrm{g} / \mathrm{ml}$. In contrast, the combination of PCZ or ICZ with either TCR or 17AAG rendered S. prolificans exquisitely more sensitive to the triazoles than did use of the triazoles alone (Table 1 ). Specifically, in combination with TCR or 17AAG, PCZ and ICZ were synergistic, with a fractional inhibitory concentra- tion index ( $(\mathrm{FIC})$ of 0.5 . In addition, bis-[1,3dibutylbarbituric acid] trimethine oxonol (DiBAC) vital staining revealed enhanced uptake of stain and plasma membrane damage in S. prolificans germlings (isolates 1 and 2) exposed to PCZ or ICZ in combination with TCR or 17AAG (Figure 1 A-C; Table S1). Use of PCZ or ICZ (0.125$0.25 \mu \mathrm{g} / \mathrm{ml}$ ) in combination with TCR or 17AAG resulted in 2.0 - to 2.5 -fold greater plasma membrane damage than did the use of triazoles alone.

Detection of intracellular Reactive Oxygen Species (ROS) accumulation and loss of mitochondrial membrane potential $\left(\Delta \Psi_{m}\right)$ in $S$. prolificans (isolates 1 and 2) germlings in response to treatment with PCZ or ICZ combined with TCR or 17AAG

Staining of $S$. prolificans germlings with dihydrorhodamine (DHR)-123 (red fluorescence) and rhodamine (Rh)-123 (green fluorescence) was most prominent in germlings treated with PCZ or ICZ in combination with TCR or 17AAG (Figures 2 and 3). A small percentage of control germlings and germlings treated with PCZ or ICZ alone exhibited positive staining for DHR-123 and Rh-123 (Figures 2 and 3). Staining with DHR123 and Rh-123 increased markedly when triazoles were combined with TCR or 17AAG, respectively (1.2-2.1 fold increase in fluorescence intensity), compared with triazoles alone (Figures 2 and $3 \mathrm{~A}-\mathrm{C}$ ). Isolate 2 in particular, had 1.0-2.1 fold and 1.3-2.1 fold increase in fluorescence for ROS accumulation and loss of mitochondrial potential, respectively, over germlings treated with triazoles alone (Table S1). Accumulation of intracellular ROS and disruption of $\Delta \Psi_{\mathrm{m}}$ are important steps in mitochondria-mediated apoptosis. These data indicate that treatment with PCZ or ICZ combined with TCR or 17AAG can trigger apoptosis in $S$. prolificans due to accumulation of ROS.

TABLE 1. In vitro antimicrobial activity of PCZ and ICZ in combination with TCR or 17AAG against S. prolificans isolates.

\begin{tabular}{|c|c|c|c|}
\hline \multirow{2}{*}{ Drugs } & \multicolumn{3}{|c|}{$\operatorname{MIC}(\mu \mathrm{g} / \mathrm{ml})$} \\
\hline & Isolate 1 & Isolate 2 & Isolate 3 \\
\hline PCZ & $128(>128) *$ & $128(>128)$ & $128(>128)$ \\
\hline ICZ & $128(>128)$ & $128(>128)$ & $128(>128)$ \\
\hline TCR & $128(>128)$ & $128(>128)$ & $128(>128)$ \\
\hline 17AAG & $32(128)$ & $64(>128)$ & $64(>128)$ \\
\hline $\begin{array}{c}\text { PCZ + TCR } \\
(0.06 \mu \mathrm{g} / \mathrm{ml})\end{array}$ & $0.50(0.50)$ & $0.25(1.00)$ & $0.25(0.50)$ \\
\hline $\begin{array}{c}\text { ICZ + TCR } \\
(0.125 \mu \mathrm{g} / \mathrm{ml})\end{array}$ & $0.25(1.00)$ & $0.25(0.50)$ & $0.25(0.50)$ \\
\hline $\mathrm{PCZ}+17 \mathrm{AAG}(0.06 \mu \mathrm{g} / \mathrm{ml})$ & $0.25(0.50)$ & $0.25(1.00)$ & $0.25(0.50)$ \\
\hline $\mathrm{ICZ}+17 \mathrm{AAG}(0.125 \mu \mathrm{g} / \mathrm{ml})$ & $0.125(0.50)$ & $0.125(0.50)$ & $0.125(0.50)$ \\
\hline
\end{tabular}

*MFC is given in parenthesis. 
A
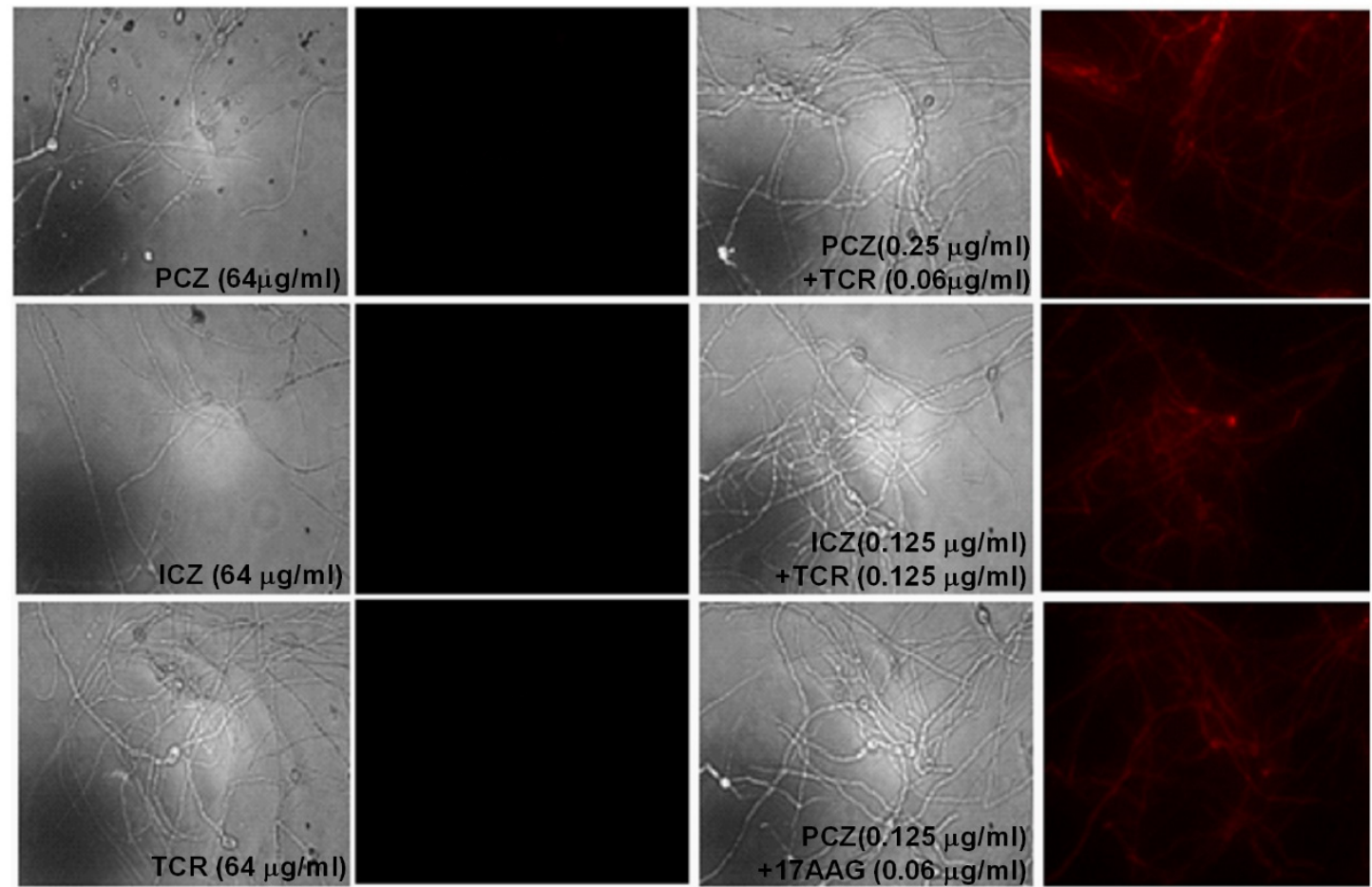

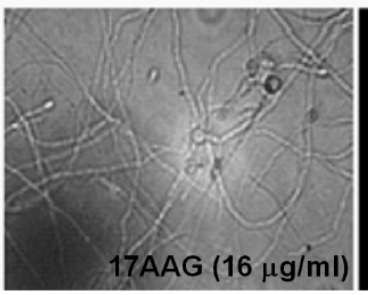

DIC

B

$$
\text { O্ঠ }
$$

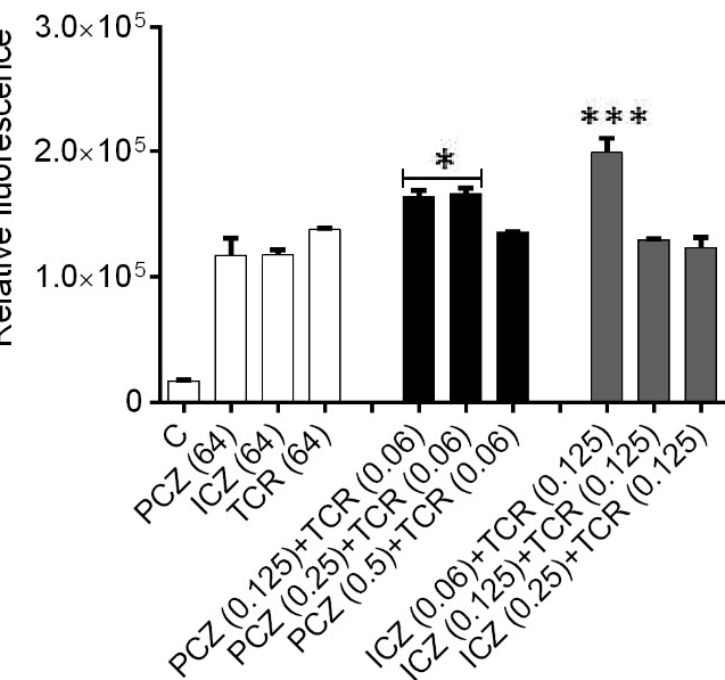

Antifungal Concentration $(\mu \mathrm{g} / \mathrm{ml})$

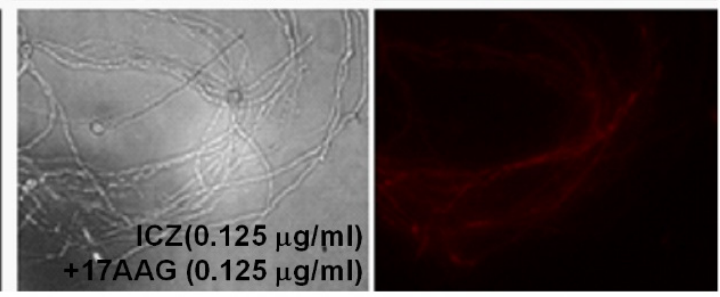

C

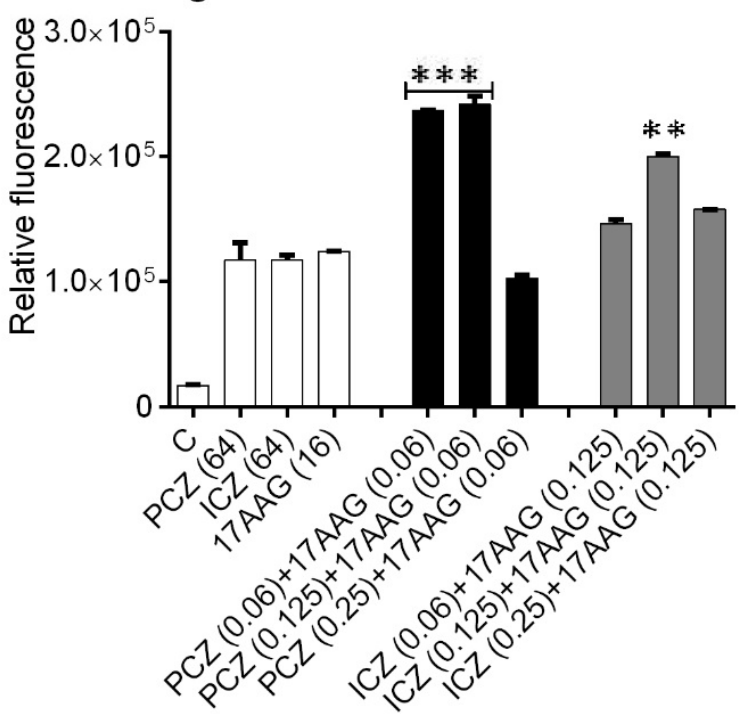

Antifungal Concentration $(\mu \mathrm{g} / \mathrm{ml})$

FIGURE 2: Intracellular ROS accumulation as detected by DHR-123 in S. prolificans isolate 1 germlings treated with PCZ or ICZ with either TCR or 17AAG, was measured using fluorescence spectrophotometry. (A) Fluorescent images of S. prolificans stained with DHR-123. (B) Relative fluorescence levels in S. prolificans germlings treated with PCZ or ICZ in combination with TCR. (C) Measurement of fluorescence of germlings treated with PCZ or ICZ in combination with 17AAG. ${ }^{*} p<0.05 ;{ }^{* *} p<0.001 ;{ }^{* *} p<0.0001$. Error bars on graphs indicate standard deviation. DIC, differential interference contrast. 
Evidence of apoptosis in S. prolificans (isolates 1 and 2) induced by treatment with PCZ or ICZ in combination with TCR or 17AAG

Because various drugs can induce both apoptosis and necrosis in mammalian cells [19], we sought to differentiate between apoptotic and necrotic $S$. prolificans protoplast using annexin V-fluorescein isothiocyanate (FITC)propidium iodide (PI) double staining, in which apoptotic cells are stained with annexin V-FITC (green), whereas the nuclei of necrotic cells are stained with PI (red) [20-22]. Incubation of $S$. prolificans (isolate 1) protoplasts in the presence of PCZ $(0.25 \mu \mathrm{g} / \mathrm{ml})$ or ICZ $(0.125 \mu \mathrm{g} / \mathrm{ml})$ in combination with TCR $(0.060-0.125 \mu \mathrm{g} / \mathrm{ml})$ at $37^{\circ} \mathrm{C}$ for $3 \mathrm{~h}$ led to annexin V-FITC staining of $35-50 \%$ of the protoplasts. We found that $30-40 \%$ of protoplasts exhibited annexin VFITC staining, when incubated with PCZ or ICZ in combination with 17AAG $(0.060-0.125 \mu \mathrm{g} / \mathrm{ml}$ ) (Table 2). In S. prolificans isolate 2 , however, $40-65 \%$ of protoplasts were apoptotic after incubation with PCZ or ICZ in combination with TCR, and $35-70 \%$ were apoptotic after incubation with PCZ or ICZ with 17AAG (Table S1). We observed no annexin VFITC staining in untreated protoplasts (Table 2). These results suggested that a fungicidal property of PCZ and ICZ was due to induction of apoptosis in S. prolificans cells, especially in combination with TCR or 17AAG.

To confirm the apoptotic features of PCZ and ICZ in S. prolificans germlings, we evaluated nuclear DNA fragmentation using a terminal deoxynucelotidyl transferase dUTP nick end labeling (TUNEL) assay. $S$. prolificans germlings exposed to PCZ or ICZ for $3 \mathrm{~h}$ at $37^{\circ} \mathrm{C}$ exhibited marked nuclear DNA fragmentation in a concentration-dependent manner (Table 2). The proportion of TUNEL-positive germlings was higher in both isolate 1 (50-60\%) and isolate $2(30-60 \%)$ in the presence of PCZ or ICZ $(0.125 \mu \mathrm{g} / \mathrm{ml})$ combined with 17AAG than when combined with TCR (isolate 1, 20-40\%; isolate 2, 35-55\%) (Tables 2 and S1).

Induction of caspase-like activity in S. prolificans (isolate 1) germlings treated with PCZ or ICZ in combination with TCR or 17AAG

Caspases are activated in the early stages of apoptosis and play a central role in the apoptotic cascade [23, 24]. Although caspases are not present in fungi, researchers have identified orthologs of mammalian caspases, called metacaspases in fungi (25). We stained S. prolificans germlings (isolate 1) pretreated with PCZ or ICZ in combination with TCR or 17AAG with the cell-permeable, broad-spectrum caspase inhibitor CaspACE-Z-VAD-FMK. In this staining, a green fluorescent signal is a direct measure of the amount of active caspase in a cell. S. prolificans germlings with activated metacaspases, treated with azoles in combination with TCR or 17 AAG were stained green, whereas germlings exposed to azoles alone remained unstained. This result indicated that treatment with PCZ or ICZ plus TCR or 17AAG triggered an apoptotic pathway in $S$. prolificans germlings via activation of metacaspases (Figure 4).
TABLE 2. Percentage of $S$. prolificans (isolate 1 ) cells stained with annexin V, TUNEL and PI for detection of phosphatidylseriene exposure, DNA fragmentation and cell membrane integrity respectively.

\begin{tabular}{|c|c|c|c|}
\hline \multirow{2}{*}{$\begin{array}{l}\text { Drugs } \\
\text { ( } \mu \mathrm{g} / \mathrm{ml})\end{array}$} & \multicolumn{3}{|c|}{ Apoptotic protoplast \% } \\
\hline & Annexin V & TUNEL & PI \\
\hline Control & - & - & $4.0 \pm 1.0$ \\
\hline PCZ (64.0) & - & - & - \\
\hline ICZ (64.0) & - & - & $5.0 \pm 1.0$ \\
\hline TCR (64.0) & - & - & $3.0 \pm 1.0$ \\
\hline 17AAG (16.0) & - & - & $3.0 \pm 1.0$ \\
\hline \multicolumn{4}{|l|}{$\begin{array}{c}\mathrm{PCZ}+\mathrm{TCR} \\
(0.06 \mu \mathrm{g} / \mathrm{ml})\end{array}$} \\
\hline 0.125 & $10.0 \pm 0.0$ & $18.0 \pm 2.0$ & - \\
\hline 0.25 & $50.0 \pm 3.0$ & $35.0 \pm 1.0$ & - \\
\hline 0.5 & $35.0 \pm 2.0$ & - & $15.0 \pm 1.0$ \\
\hline \multicolumn{4}{|l|}{$\begin{array}{c}\text { ICZ + TCR } \\
(0.125 \mu \mathrm{g} / \mathrm{ml})\end{array}$} \\
\hline 0.060 & $23.0 \pm 2.0$ & $12.0 \pm 1.0$ & $3.0 \pm 1.0$ \\
\hline 0.125 & $30.0 \pm 1.0$ & $35.0 \pm 1.0$ & $5.0 \pm 1.0$ \\
\hline 0.25 & $40.0 \pm 2.0$ & $40.0 \pm 2.0$ & $15.0 \pm 1.0$ \\
\hline \multicolumn{4}{|l|}{$\begin{array}{l}\text { PCZ + 17AAG } \\
(0.06 \mu \mathrm{g} / \mathrm{ml})\end{array}$} \\
\hline 0.060 & $10.0 \pm 1.0$ & $12.0 \pm 1.0$ & - \\
\hline 0.125 & $35.0 \pm 3.0$ & $60.0 \pm 4.0$ & $3.0 \pm 1.0$ \\
\hline 0.25 & $40.0 \pm 2.0$ & $22.0 \pm 1.0$ & $12.0 \pm 2.0$ \\
\hline \multicolumn{4}{|l|}{$\begin{array}{l}\text { ICZ + 17AAG } \\
(0.125 \mu \mathrm{g} / \mathrm{ml})\end{array}$} \\
\hline 0.060 & $5.0 \pm 1.0$ & $20.0 \pm 1.0$ & - \\
\hline 0.125 & $30.0 \pm 1.0$ & $50.0 \pm 4.0$ & $5.0 \pm 0.0$ \\
\hline 0.25 & $30.0 \pm 2.0$ & $10.0 \pm 1.0$ & $15.0 \pm 0.0$ \\
\hline
\end{tabular}

*MFC is given in parenthesis.

\section{DISCUSSION}

We hypothesized that TCR and 17AAG enhance the negligible activity of the ergosterol biosynthesis inhibitors PCZ and ICZ, to the point that they become fungicidal, and that this fungicidal activity is mediated through apoptosis in $S$. prolificans. The calcineurin pathway and $\mathrm{Hsp90}$ are important for the survival of pathogenic fungi because they 
A
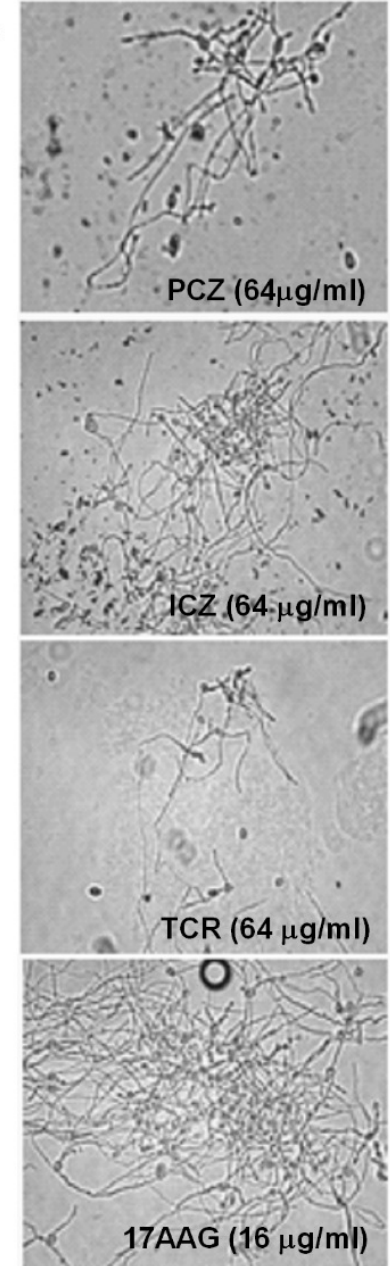

DIC

B

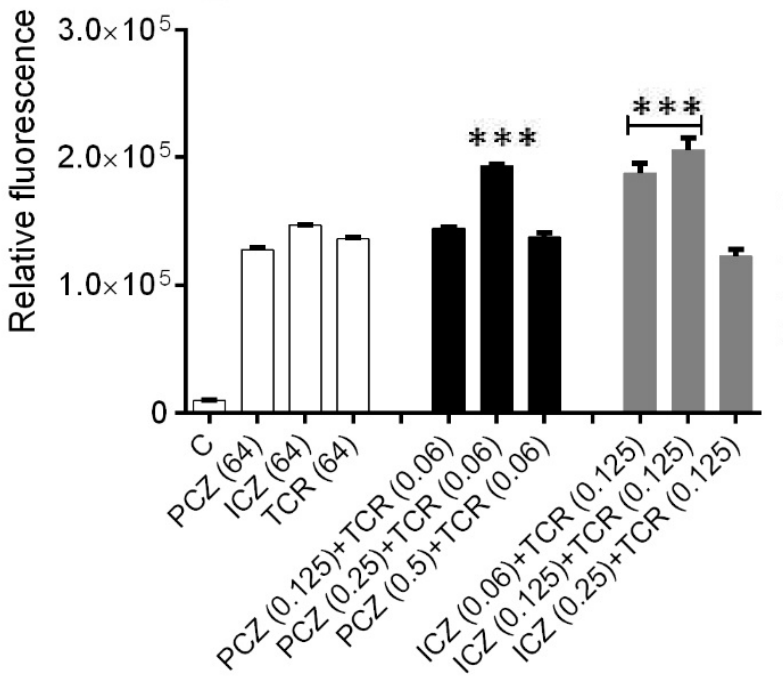

Antifungal Concentration ( $\mu \mathrm{g} / \mathrm{ml})$

Rh123
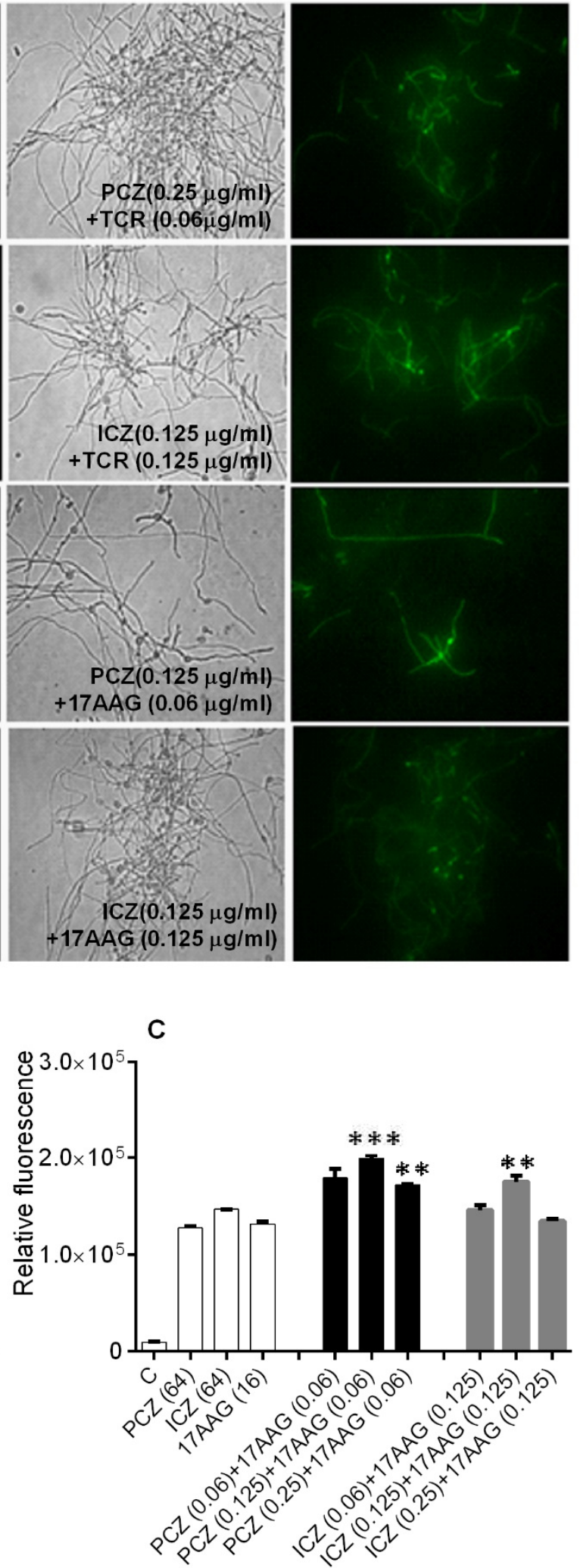

Antifungal Concentration $(\mu \mathrm{g} / \mathrm{ml})$

FIGURE 3: Changes in $\Delta \Psi_{\mathrm{m}}$ in S. prolificans isolate 1 germlings triggered by treatment with PCZ or ICZ combined with TCR or 17AAG. (A) Fluorescence images of $S$. prolificans germlings stained with Rh-123. (B, C) Relative fluorescence levels in $S$. prolificans germlings treated with PCZ or ICZ plus TCR (B) and PCZ or ICZ plus 17AAG (C). ${ }^{* *} p<0.001 ;{ }^{* *} \mathrm{p}<0.0001$. Error bars on graphs indicate standard deviation. DIC, differential interference contrast. 
have central roles in various cellular processes, including morphogenetic transition and development of antifungal tolerance and resistance $[7,16]$. Inhibition of the calcineurin pathway and Hsp90 in combination with administration of conventional antifungal agents may have broad therapeutic potential in patients with fungal infections $[16,26]$. Owing to the immunosuppressive properties of calcineurin inhibitors and the role of Hsp90 in controlling the calcineurin pathway, clinical use of a combination of TCR or 17AAG with triazole for treatment of $S$. prolificans infection would ultimately require a novel antifungal agent that selectively targets fungal stress pathways without having collateral effects on human immune cells.

We found evidence of synergy of PCZ and ICZ with TCR and $17 A A G$ in $S$. prolificans in vitro, which is consistent with data on other fungal species $[12,16,18,21]$. In addition, we used multiple markers of cell death to show that apoptosis is a mechanism of PCZ/ICZ- and TCR/17AAGinduced cell death. We corroborated the rate of apoptosis in S. prolificans germlings using assays for detection of phosphatidylserine (PS) by annexin V-FITC, ROS accumulation by DHR-123 staining and decreased mitochondrial membrane potential by Rh123, DNA damage by TUNEL staining, and activation of caspase-like activity by CaspACE FITC-VAD-FMK. In each of the assays, apoptosis was evident at PCZ, ICZ, TCR, and 17AAG concentrations (0.125$0.250 \mu \mathrm{g} / \mathrm{ml}$ ) that were below the MIC of triazoles. Taken together, these data indicate that PCZ or ICZ combined with TCR or 17AAG at concentrations below the MIC causes apoptosis in S. prolificans germlings.

We found that induction of apoptosis and the fungicidal activity of PCZ and ICZ in combination with TCR or 17AAG correlated with increased plasma and mitochondrial membrane disruption, PS externalization, DNA fragmentation, and ROS accumulation in $S$. prolificans germlings (isolates 1 and 2) (Tables 1, 2 and S1, Figures 1-4). Calcineurin activity is known to contribute to the fungicidal effects of Hsp90 inhibitors. 17-AAG in particular induces
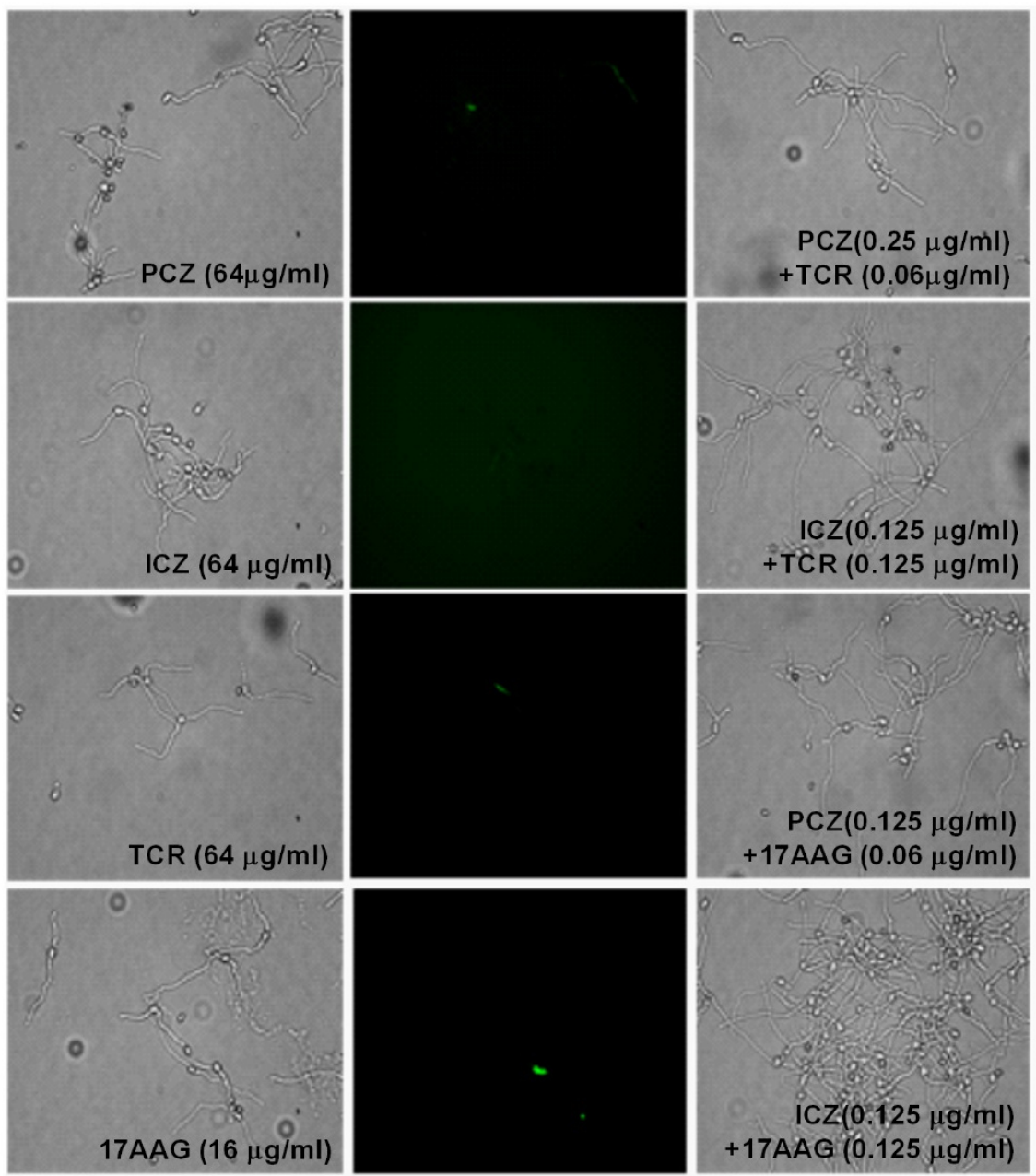
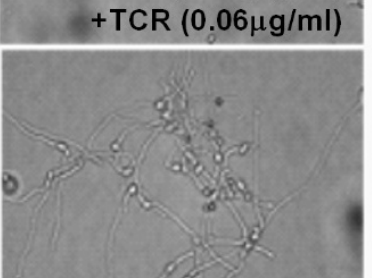

ICZ $(0.125 \mu \mathrm{g} / \mathrm{ml})$ +TCR $(0.125 \mu \mathrm{g} / \mathrm{ml})$
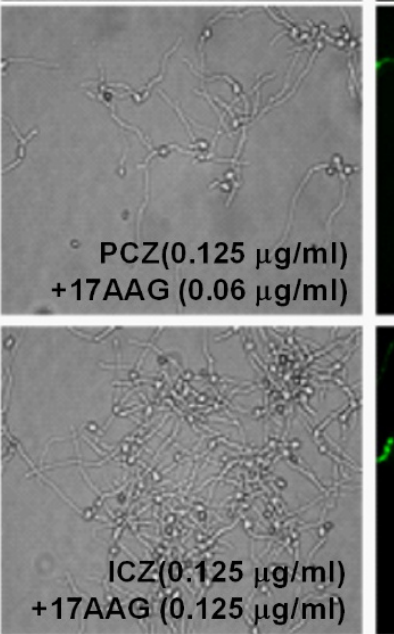
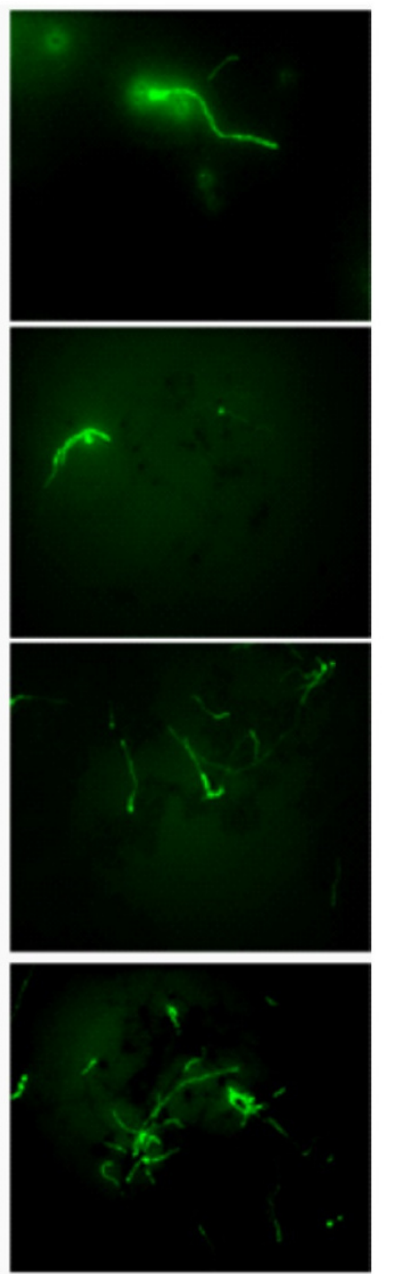

FIGURE 4: Detection of metacaspase (caspase-like) activity using CaspACE FITC-VAD-FMK probe in germlings of $S$. prolificans (isolate 1) treated with PCZ or ICZ in combination with TCR or 17AAG. Shown are fluorescent images of activated metacaspases of S. prolificans germlings treated with drugs alone and in combination with TCR or 17AAG. 
apoptosis in colon carcinoma-derived cell lines (27), so determination of whether inhibition of $\mathrm{Hsp} 90$ can induce apoptotic cell death in fungi would be of interest. Dai et al. [28] demonstrated the role of Hsp90 in apoptosis in C. albicans and showed that inhibition of Hsp90 attenuated apoptosis by regulating the calcineurin pathway. Several fungi undergo apoptosis in response to antifungal treatment and various other stimuli [19]. Additional studies providing better understanding of fungal apoptotic pathways would promote the discovery of much-needed antifungal therapies.

Our results indicated that disruption of mitochondrial integrity by a combination of PCZ/ICZ with TCR or 17AAG induced apoptosis in S. prolificans. Our study in S. prolificans and studies in other fungi showed that 17AAG inhibits Hsp90, causing mitochondria-mediated apoptosis in rat histiocytomas [29]. Also, Shirazi and Kontoyiannis [18] showed that increased apoptosis after exposure to TCR was correlated with increased intracellular ROS accumulation in Mucorales. Furthermore, translocation of mitochondrial cyt $c$ to the cytosol has led to binding of cyt $c$ with apoptotic protease-activating factor to form a complex with caspase-9, resulting in caspase activation $[23,24$, 30]. Release of cyt $c$ requires an increase in mitochondrial membrane permeability during apoptosis [23]. As in Mucorales, our results in S. prolificans also demonstrated that, ROS formation, changes in $\Delta \Psi_{\mathrm{m}}$, and cyt $c$ release were associated with apoptosis [20-22]. Authors have also reported ROS-induced apoptosis in A. nidulans, Fusarium oxysporum, and C. albicans [31-33]. Sharon et al. [25] reported that apoptotic pathways in fungi seem to be mitochondrion-dependent, and can be powerful sources of superoxide radicals in cells undergoing miconazole and farnesol-induced apoptosis [34].

Authors have reported accumulating evidence that different stimuli induce different apoptotic pathways in yeasts and other fungi $[35,36]$. In mammals, apoptosis is regulated by activation of caspases, which cleave specific substrates and trigger apoptotic death [37]. Now it is evident that caspase-like proteolytic activity may exist not only in multicellular organisms but also unicellular organisms, such as fungi. In the current study, we observed caspase-like activity in S. prolificans germlings upon exposure to PCZ or ICZ with TCR or 17AAG. Further studies are needed to demonstrate how proteases contribute to apoptotic fungal death.

In conclusion, we have shown for the first time that coadministration of inhibitors of the ergosterol biosynthesis pathways with an inhibitor of calcineurin or Hsp90 induces apoptosis in the recalcitrant fungus S. prolificans. This fungicidal synergistic interaction requires further study, as it may be a useful therapeutic strategy for infections caused by pathogenic fungi for which treatment options are extremely limited.

\section{MATERIALS AND METHODS \\ Drugs}

PCZ stock (5 mg/ml; Merck \& Co., Inc.) was prepared in distilled water. ICZ (5 mg/ml; Janssen Pharmaceuticals), TCR (1 $\mathrm{mg} / \mathrm{ml}$; Medisca), and 17AAG (Sigma) stocks were prepared in ethanol, and aliquots were stored at $-20^{\circ} \mathrm{C}$ in the dark until use.

\section{Isolates and growth conditions}

Three clinical isolates of S. prolificans (S.p-071507 [isolate 1], 071826 [isolate 2], and 674802 [isolate 3]) were grown on freshly prepared Sabouraud dextrose agar plates. After $48 \mathrm{~h}$ of incubation at $37^{\circ} \mathrm{C}$, spores were collected and washed twice in sterile phosphate-buffered saline (PBS). The spores were then counted using a hemocytometer and stored at $4^{\circ} \mathrm{C}$ in PBS.

\section{Susceptibility testing}

Broth microdilution was performed according to the Clinical and Laboratory Standards Institute method [38]. Briefly, twofold serial PCZ and ICZ dilutions were prepared in flatbottomed 96 -well microtiter plates $(100 \mu \mathrm{l} /$ well) in the presence or absence of TCR or 17AAG $(0.060-0.125 \mu \mathrm{g} / \mathrm{ml})$. Drugfree wells were used as controls. Each well was inoculated with $100 \mu \mathrm{l}$ of freshly isolated S. prolificans spores (2-3 days old; $1 \times 10^{4}$ spores $/ \mathrm{ml}$ ) suspended in RPMI medium. After $48 \mathrm{~h}$ of incubation at $37^{\circ} \mathrm{C}$, the MICs of PCZ and ICZ were determined visually as the lowest drug concentrations resulting in complete growth inhibition. To determine the minimum fungicidal concentrations (MFC) of PCZ and ICZ, an aliquot (20 $\mu$ l) from each well that exhibited $100 \%$ growth inhibition was plated onto YPD agar ( $1 \%$ yeast extract, $2 \%$ peptone, $2 \%$ dextrose and $2 \%$ agar) plates. After $24 \mathrm{~h}$ of incubation at $37^{\circ} \mathrm{C}$, the MFC was recorded as the lowest drug concentration at which no growth was observed.

For all of the wells of the microtiter plates that corresponded to MICs, the sum of the fractional inhibitory concentrations $(\Sigma F I C)$ was calculated for each well using the equation $\Sigma F I C=$ FICA + FICB = $(C A / M I C A)+(C B / M I C B)$, in which MICA and $M I C B$ are the MICs of drugs $A$ and $B$ alone, respectively, and $C A$ and $C B$ are the concentrations of the drugs in combination, respectively, in all of the wells corresponding to an MIC. Synergy was defined as a $\Sigma F I C$ of up to 0.5 . Indifference was defined as a $\Sigma$ FIC of at least 0.5 but no more than 4.0. Antagonism was defined as a $\Sigma$ FIC greater than 4.0.

\section{Viability assay}

S. prolificans germlings (isolates 1 and 2) treated with TCR or 17AAG $(0.060-0.125 \mu \mathrm{g} / \mathrm{ml})$ along with PCZ $(0.06-0.50 \mu \mathrm{g} / \mathrm{ml})$ or ICZ $(0.06-0.25 \mu \mathrm{g} / \mathrm{ml})$ for $3 \mathrm{~h}$ were stained with DiBAC (Molecular Probes) as described previously [21, 22].

\section{Annexin V-FITC-PI double staining of S. prolificans (isolates 1 and 2)}

The apoptosis marker PS is located on the inner leaflet of the lipid bilayer of the cytoplasmic membrane and is translocated to the outer leaflet at the onset of apoptosis [39-41]. PS can be detected using staining with annexin V-FITC, which binds to it. Germlings treated with PCZ $(0.06-0.50 \mu \mathrm{g} / \mathrm{ml})$ or ICZ $(0.06$ $0.25 \mu \mathrm{g} / \mathrm{ml})$ in combination with TCR or 17AAG $(0.060$ and $0.125 \mu \mathrm{g} / \mathrm{ml}$ ) were digested with a lysing enzyme mixture $(0.25 \mathrm{mg} / \mathrm{ml}$ chitinase, $15 \mathrm{U}$ of lyticase, and $20 \mathrm{mg} / \mathrm{ml}$ lysing enzyme; Sigma) for $3 \mathrm{~h}$ at $30^{\circ} \mathrm{C}$. After digestion, S. prolificans protoplasts were stained with annexin V-FITC (BD Pharmingen) and $\mathrm{Pl}$ at room temperature for $15 \mathrm{~min}$ and ob- 
served under a fluorescence microscope to assess the externalization of PS as described previously [39].

Detection of intracellular ROS accumulation and $\Delta \Psi_{m}$ in $S$. prolificans germlings (isolates 1and 2)

ROS plays an important role as an early initiator of apoptosis in yeasts and other filamentous fungi [20-22]. The amount of ROS in S. prolificans germlings was measured using DHR-123 (Sigma) staining [20-22]. The mitochondrial membrane potential was assessed by staining with Rh-123 (Sigma), a fluorescent dye that diffuses in the matrix in response to electric potential as described [20-22]. Intracellular ROS levels and $\Delta \Psi_{\mathrm{m}}$ in $S$. prolificans germlings were measured after treatment with PCZ $(0.060-0.50 \mu \mathrm{g} / \mathrm{ml})$ or ICZ $(0.060-0.25 \mu \mathrm{g} / \mathrm{ml})$ in combination with TCR and 17 AAG $(0.060$ and $0.125 \mu \mathrm{g} / \mathrm{ml})$ for $3 \mathrm{~h}$ at $37^{\circ} \mathrm{C}$ using a fluorimetric assay with DHR-123 and Rh123 staining [20-22, 42].

\section{Measurement of DNA damage in S. prolificans (isolates 1 and} 2)

DNA fragmentation, a characteristic of apoptosis, was detected in S. prolificans using a TUNEL assay. Germlings pretreated with PCZ $(0.06-0.50 \mu \mathrm{g} / \mathrm{ml})$ or ICZ $(0.06-0.25 \mu \mathrm{g} / \mathrm{ml})$ in combination with TCR or 17AAG $(0.060$ and $0.125 \mu \mathrm{g} / \mathrm{ml})$ for $3 \mathrm{~h}$ at $37^{\circ} \mathrm{C}$ were fixed with $3.7 \%$ formaldehyde for $30 \mathrm{~min}$ on ice and digested using a lysing enzyme mixture. Enzyme-digested germlings were used to detect DNA fragmentation using a TUNEL assay as described by Madeo et al. [41]. The protoplasts were observed for fluorescence with excitation and emission wavelengths of $488 \mathrm{~nm}$ and $520 \mathrm{~nm}$, respectively.

Detection of metacaspase activity using CaspACE FITC-VADFMK in S. prolificans germlings (isolates 1 and 2)

Active metacaspases in S. prolificans germlings were detected using CaspACE FITC-VAD-FMK (Promega) according to the manufacturer's instructions [20-22]. Briefly, germlings pretreated with PCZ $(0.06-0.50 \mu \mathrm{g} / \mathrm{ml})$ or ICZ $(0.06-0.25 \mu \mathrm{g} / \mathrm{ml})$ in combination with TCR or 17AAG $(0.060$ and $0.125 \mu \mathrm{g} / \mathrm{ml})$ for 3 $\mathrm{h}$ at $37^{\circ} \mathrm{C}$ were collected, washed in PBS, resuspended in 10 $\mu \mathrm{M}$ FITC-VAD-FMK, and incubated again for $2 \mathrm{~h}$ at $30^{\circ} \mathrm{C}$. Apoptosis in the $S$. prolificans germlings was inhibited in the presence of the caspase inhibitor Z-VAD-FMK (Sigma) at final concentrations of $40 \mu \mathrm{M}$. After incubation, germlings were

\section{REFERENCES}

1. Gosbell IB, Morris ML, Gallo JH, Weeks KA, Neville S, Rogers AH, Andrews RH, and Ellis DH (1999). Clinical, pathologic and epidemiologic features of infection with Scedosporium prolificans: four cases and review. Clin Microbiol Infect 5:672-686.

2. Cuenca-Estrella M, Ruiz-Diez B, Martinez-Suarez JV, Monzon A, and Rodriguez-Tudela JL (1999). Comparative in-vitro activity of voriconazole $(U K-109,496)$ and six other antifungal agents against clinical isolates of Scedosporium prolificans and Scedosporium apiospermum. J Antimicrob Chemother 43:149-151.

3. Cortez KJ, Roilides E, Quiroz-Telles F, Meletiadis J, Antachopoulos C, Knudsen T, Buchanan W, Milanovich J, Sutton DA, Fothergill A, Rinaldi MG, Shea YR, Zaoutis T, Kottilil S, and Walsh TJ (2008). Infections caused by Scedosporium spp. Clin Microbiol Rev 21: 157e197. washed twice in PBS and observed microscopically for fluorescence with excitation and emission settings of $488 \mathrm{~nm}$ and 520 $\mathrm{nm}$, respectively.

\section{Statistical Analysis}

For all assays, three independent experiments were performed in triplicate. Comparisons of multiple treatment groups were performed by using two-way analysis of variance with post-hoc paired comparisons using Dunnett's test. Calculations were made using the InStat software program (GraphPad Software). Two-tailed $P$ values of less than 0.05 were considered statistically significant.

\section{ACKNOWLEDGMENTS}

D.P.K. acknowledges the Frances King Black Endowed Professorship for Cancer Research. This research was supported in part by the National Institutes of Health through MD Anderson's Cancer Center Support Grant P30CA016672.

\section{SUPPLEMENTAL MATERIAL}

All supplemental data for this article are available online at www.microbialcell.com.

\section{CONFLICT OF INTEREST}

D.P.K has received research support and honoraria from Pfizer, Astellas Pharma US, and Merck and Co. Inc, F.S reports no conflicts.

\section{COPYRIGHT}

(C) 2014 Shirazi and Kontoyiannis. This is an open-access article released under the terms of the Creative Commons Attribution (CC BY) license, which allows the unrestricted use, distribution, and reproduction in any medium, provided the original author and source are acknowledged.

Please cite this article as: Fazal Shirazi and Dimitrios P. Kontoyiannis (2014). Heat shock protein 90 and calcineurin pathway inhibitors enhance the efficacy of triazoles against Scedosporium prolificans via induction of apoptosis. Microbial Cell 1(6): 179-188. doi: 10.15698/mic2014.06.150

4. Lamaris GA, Chamilos G, Lewis RE, Safdar A, Raad I, and Kontoyiannis DP (2006). Scedosporium infection in a tertiary care cancer center: a review of 25 cases from 1989-2006. Clin Infect Dis 43: 1580-1584.

5. Bader T, Schroppel K, Bentink S, Aqabian N, Kohler G, and Morschhauser $J$ (2006). Role of calcineurin in stress resistance, morphogenesis, and virulence of a Candida albicans wild-type strain. Infect Immun 74: 4366-4369.

6. Cowen LE, and Lindquist S (2005). Hsp90 potentiates the rapid evolution of new traits: drug resistance in diverse fungi. Science 309: 2185-2189.

7. Cowen LE (2008). The evolution of fungal drug resistance: modulating the trajectory from genotype to phenotype. Nat Rev Microbiol 6: 187-198. 
8. Reedy JL, Filler SG, and Heitman J (2010). Elucidating the Candida albicans calcineurin signaling cascade controlling stress response and virulence. Fungal Genet Biol 47: 107-116.

9. Steinbach WJ, Cramer RA, Perfect BZ, Asfaw YG, Sauer TC, Najvar LK, Kirkpatrick WR, Patterson TF, Benjamin DK, Heitman J, and Perfect JR (2006). Calcineurin controls growth, morphology, and pathogenicity in Aspergillus fumigatus. Eukaryot Cell 5: 1091-1103.

10. Wandinger SK, Richter K, and Buchner J (2008). The Hsp90 chaperone machinery. J Biol Chem 283: 18473-18477.

11. Kahan BD (2003). Timeline: Individuality: the barrier to optimal immunosuppression. Nat Rev Immunol 3: 831-838.

12. Dannaoui E, Afeltra J, Meis JF, and Verweij PE (2002). In vitro susceptibilities of zygomycetes to combinations of antimicrobial agents. Antimicrob Agents Chemother 46: 2708-2711.

13. Kontoyiannis DP, Lewis RE, Osherov N, Albert ND, and May GS (2003). Combination of caspofungin with inhibitors of the calcineurin pathway attenuates growth in vitro in Aspergillus species. J Antimicrob Chemother 51: 313-316.

14. Narreddy S, Manavathu E, Chandrasekar PH, Alangaden GJ, and Revankar SG (2010). In vitro interaction of posaconazole with calcineurin inhibitors and sirolimus against zygomycetes. J Antimicrob Chemother 65: 701-703.

15. Lewis RE, Ben-Ami R, Best L, Albert N, Walsh TJ, and Kontoyiannis DP (2012). Tacrolimus enhances the potency of posaconazole against Rhizopus oryzae in vitro and in an experimental model of mucormycosis. J Infect Dis 5: 834-841.

16. Cowen LE, Singh SD, Kohler JR, Collins C, Zaas AK, Schell WA, Aziz H, Mylonakis E, Perfect JR, Whitesell L, and Lindquist S (2009). Harnessing $\mathrm{Hsp} 90$ function as a powerful, broadly effective therapeutic strategy for fungal infectious disease. Proc Natl Acad Sci U S A 106: 28182823.

17. Lu H, Zhu Z, Dong L, Jia X, Sun X, Yan L, Chai Y, Jiang Y, and Cao $Y$ (2011). Lack of trehalose accelerates $\mathrm{H}_{2} \mathrm{O}_{2}$-induced Candida albicans apoptosis through regulating $\mathrm{Ca}^{2+}$ signaling pathway and caspase activity. PLoS One 6(1): e15808.

18. Shirazi F, and Kontoyiannis DP (2013). The calcineurin pathway inhibitor tacrolimus enhances the in vitro activity of azoles against Mucorales via apoptosis. Eukaryot Cell 12 (9): 1225-1234.

19. Ramsdale M (2008). Programmed cell death in pathogenic fungi. Biochim Biophys Acta 1783: 1369-1380.

20. Barbu EM, Shirazi F, McGrath DM, Albert N, Sidman RL, Pasqualini $\mathrm{R}$, Arap W, and Kontoyiannis DP (2013). An antimicrobial peptidomimetic induces Mucorales cell death through mitochondriamediated apoptosis. PLoS One 8 (10): e76981.

21. Shirazi F, and Kontoyiannis DP (2013). Mitochondrial respiratory pathways inhibition in Rhizopus oryzae potentiates activity of posaconazole and itraconazole via apoptosis. PLoS One 8 (5): e63393.

22. Shirazi F, Pontikos MA, Walsh TJ, Albert N, Lewis RE, and Kontoyiannis DP (2013). Hyperthermia sensitizes Rhizopus oryzae to posaconazole and itraconazole action through apoptosis. Antimicrob Agents Chemother 57 (9): 4360-4368.

23. Wu XZ, Chang WQ, Cheng AX, Sun LM, and Lou HX (2010). Plagiochin $E$, an antifungal active macrocyclic bis(bibenzyl), induced apoptosis in Candida albicans through a metacaspase-dependent apoptotic pathway. Biochim Biophys Acta 1800: 439-447.

24. Cho J, and Lee DG (2011). The antimicrobial peptide arenicin-1 promotes generation of reactive oxygen species and induction of apoptosis. Biochim Biophys Acta 1810: 1246-1251.
25. Sharon A, Finkelstein A, Shlezinger N, and Hatam I (2009). Fungal apoptosis: function, genes and gene function. FEMS Microbiol Rev 33: 833-854.

26. LaFayette SL, Collins C, Zaas AK, Schell WA, Betancourt-Quiroz M, Gunatilaka AA, Perfect JR, and Cowen LE (2010). PKC signaling regulates drug resistance of the fungal pathogen Candida albicans via circuitry comprised of Mkc1, calcineurin, and Hsp90. PLoS Pathog 6: e1001069.

27. Hostein I, Robertson D, DiStefano F, Workman P, and Clarke PA (2001). Inhibition of signal transduction by the Hsp90 inhibitor 17allylamino-17-demethoxygeldanamycin results in cytostasis and apoptosis. Cancer Res 61: 4003-4009.

28. Dai B, Wang Y, Li D, Xu Y, Liang R, Zhao LX, CaoYB, Jia JH, and Jiang YY (2012). Hsp90 is involved in apoptosis of Candida albicans by regulating the calcineurin-caspase apoptotic pathway. PLoS One 7: e45109.

29. Taiyab A, Sreedhar AS, and Rao CM (2009). Hsp90 inhibitors, GA and 17AAG, lead to ER stress-induced apoptosis in rat histiocytoma. Biochem Pharmacol 78: 142-152.

30. Cho J, and Lee DG (2011). Oxidative stress by antimicrobial peptide pleurocidin triggers apoptosis in Candida albicans. Biochemie 93: 1873-1879.

31. Semighini CP, Hornby JM, Dumitru R, Nickerson KW, and Harris SD (2006). Farnesol-induced apoptosis in Aspergillus nidulans reveals a possible mechanism for antagonistic interactions between fungi. Mol Microbiol 59: 753-764.

32. Semighini CP, Murray N, and Harris SD (2008). Inhibition of Fusarium graminearum growth and development by farnesol. FEMS Microbiol Lett 279: 259-264.

33. Shirtliff ME, Krom BP, Meijering RA, Peters BM, Zhu J, Scheper MA, Harris ML, and Jabra-Rizk MA (2009). Farnesol-induced apoptosis in Candida albicans. Antimicrob Agents Chemother 53: 2392-2401.

34. Kobayashi D, Kondo K, Uehara N, Otokozawa S, Tsuji N, Yagihashi $A$, and Watanabe $N$ (2002). Endogenous reactive oxygen species is an important mediator of miconazole antifungal effect. Antimicrob Agents Chemother 46: 3113-3117.

35. Carmona-Gutierrez D, Eisenberg T, Buttner S, Meisinger C, Kroemer $G$, and Madeo F (2010). Apoptosis in yeast: triggers, pathways, subroutines. Cell Death Differ 17: 763-773.

36. Hamann A, Brust D, and Osiewacz HD (2008). Apoptosis pathways in fungal growth, development and ageing. Trends Microbiol 16: 276283.

37. Hengartner MO (2001). Apoptosis. DNA destroyers. Nature 412: 27-29.

38. Clinical and Laboratory Standard Institute. (2008). Reference method for broth dilution Antifungal Susceptibility Testing of filamentous fungi. Approved standard. 2nd edition. M38-A2, CLSI, Wayne, PA, USA.

39. Madeo F, Frohlich E, and Frohlich KU (1997). A yeast mutant showing diagnostic markers of early and late apoptosis. J Cell Biol 139: 729-734.

40. Madeo F, Frohlich E, Ligr M, Grey M, Sigrist SJ, Wolf DH, and Frohlich KU (1999). Oxygen stress: a regulator of apoptosis in yeast. J Cell Biol 145: 757-767.

41. Madeo F, Herker E, Maldener C, Wissing S, Lachelt S, Herlan M, Fehr M, Lauber K, Sigrist SJ, Wesselberg S, and Frohlich KU (2002). A caspase-related protease regulates apoptosis in yeast. Mol Cell 9: 911-917. 
42. Wu XZ, Cheng AX, Sun LM, Sun SJ, and Lou HX (2009). Plagiochin E, an antifungal bis(bibenzyl), exerts its antifungal activity through mitochondrial dysfunction-induced reactive oxygen species accumulation in Candida albicans. Biochim Biophys Acta 1790: 770-777. 